(Physiologisches Laboratorium in Bonn.)

\title{
Ueber die Einwirkung verdünnter Kalilauge auf Glykogen bei $100^{\circ} \mathrm{C}$.
}

Von

E. Pfiüger.

Ich babe bewiesen ${ }^{1}$ ), dass Glykogen beim Erhitzen auf $100^{\circ} \mathrm{C}$. durch Kalilauge von hoher Concentration nicht zersetzt wird.

Man hatte ja bisher auf Grund der Versuche von v. Vintschga und Dietl ${ }^{2}$ ) u. s. w. als sicher angenommen, dass eine Glykogenlösung durch Kalilauge zerstört werde. Diese Versuche sind aber immer nur mit verdünnter, d. h. 1 bis $2 \%$ iger Kalilauge ausgeführt worden. Fs musste desshalb die Möglichkeit geprüft werden, ob diese verdünnte Kalilauge beim Kochen das Glykogen zersetzt.

Wie ich bereits vor Kurzem ${ }^{2}$ ) berichtet habe, erhielt ich in der That in einer Versuchsreihe das Ergebniss, „dass Glykogen, welches „mit den Brü cke' schen Reagentien in keine Berührung gekommen „war, zwar nicht von concentrirter, wohl aber von verdünnter "(2\% iger) Kalilauge in geringem Grade beim längeren Kochen an"gegriffen wurde. Der Verlust betrug $3,9 \%$. "

Weil ich einen Beobachtungsfehler vermuthete, versprach ich, den Versuch sofort zu wiederholen und darüber zu berichten. Ich löse dies Versprechen hiermit ein. -

Um vollste Sicherheit zu erzielen, wurde bei der Gewinnung des Glykogenes die Anwendung jeder Säure sowie der Brücke'schen Reagentien ausgeschlossen.

Zuerst prüfte ich, ob mein Filtrirpapier etwa, wenn es wie bei einem Versuche benutzt wird, Kohlehydrate abgibt. Ich untersuchte also mein schwedisches Filtrirpapier. Es stammt laut Aufschrift der

1) Dieses Archiv Bd. 90 S. 528 (9. Juni 1902); dieses Archiv Bd. 91 S. 119 (1902).

2) Dieses Archiv Bd. 13 S. 253 und Bd. 17 S. 154.

3) Dieses Archiv Ba. 92 S. 101 (1902).

E. Pflîger, Archiv fúr Physiologie. Ba. 93. 
Packete aus J. H. Munktell's Fabrik. Meine Filter haben den Durchmesser von $15 \mathrm{~cm}$ und zeichnen sich dadurch aus, dass sie alkalischen Laugen von beträchtlicher Stärke widerstehen.

Ich nahm $400 \mathrm{ccm}$ Kalilauge von $15 \% \mathrm{KOH}+400 \mathrm{ccm}$ Alkohol von $96 \%$ Tr.; eine Mischung, welche beim Abfiltriren des Glykogenes öfter gebraucht wird. Ich filtrirte diese $800 \mathrm{ccm}$ Lösung, welche vollkommen klar war, durch das schwedische Filter. Die Filtration vollzog sich auffallend langsam und nahm viele Stunden in Anspruch. Nachdem vollkommen abgetropft war, steckte ich den Trichter auf ein $300 \mathrm{ccm}-K o ̈ l b c h e n$ und goss $100 \mathrm{cem} 2,2 \% \mathrm{ClH}$ auf. Das Filtrat war bereits scharf sauer; dann wusch ich das Filter mit noch ungefähbr $200 \mathrm{cem}$ ClH von 2,2\% aus. Als das $300 \mathrm{ccm}-K o ̈ l b c h e n$ annähernd gefullt war, wurde es zur Invertirung im Wasserbad 3 Stunden erhitzt. Ich verfuhr sodann wie bei einer Zuckeranalyse nach Pflüger. Es war aber keine Spur einer Abscheidung von Kupferoxydul zu sehen, obwohl ich die blaue Flüssigkeit 24 Stunden bedeckt im Becherglas stehen liess. Unter beschriebener Bedingung unterliegt es also keinem Bedenken, die schwedischen Filter zu benutzen. -

Wenn der Organbrei in $30 \%$ iger Kalilauge von $100^{\circ}$ in Lösung gebracht ist, verdüne ich auf das doppelte Volum, so dass ich eine Flüssigkeit mit $15 \% \mathrm{KOH}$ erhalte, welche filtrirt werden muss, weil ștets Flöckchen vorhanden sind. Am bequemsten wären die gehärteten grossen Filter von Schleicher \& Schüll Nr. 575 von $32 \mathrm{~cm}$ Durchmesser. Weil hier das gelöste Glykogen in das Filtrat übergeht, fragt es sich, ob die alkalische Lösung ein Kohlehydrat àu dem Papier mitnimmt. Ich nahm also $400 \mathrm{ccm}$ einer wässerigen Lauge von $15 \% \mathrm{KOH}$ und filtrirte sie drei Mal durch das Filter und versetzte daun das vollkommen klare Filtrat mit $400 \mathrm{ccm} \mathrm{Al-}$ kohol von $96 \% \mathrm{Tr}$. Nach einigen Stunden hatten sich einige spärliche, leichte, durchsichtige, farblose Wölkchen ausgeschieden.

Ich filtrirte dieselben nach 24 Stunden durch das schwedische 1 cm-Filter $a b$, liess gut abtropfen und spülte dann, wie oben berichtet, das Filter mit $2,2 \% \mathrm{ClH}$ so aus, dass das saure Filtrat in ein $300 \mathrm{ccm}-K o ̈ l b c h e n$ floss und dieses beinahe bis zur Marke gefüllt war. Nach Inversion untersuchte ich den Gehalt an Zucker, wie es bei einer Glykogenbestimmung von mir stets ansgeführt wird. Unmittelbar nach der 1/2 stündigen Erhitzung der All ị h n'schen Kupferlösung war von Kupferoxydul nichts zu sehen. Als ich aber nach 24 stündigem 
Stehen untersuchte, konnte ich auf dem Boden einen feinen Staub erkennen, tër nach dem vorsichtigen Abgiessen der blauen Flüssigkeit sich als ein rothes Pulver darstellte, welches ein wenig mehr war, als man wohl unter Umständen durch die Selbstreduction entstanden wahrnimmt ${ }^{1}$ ). Wenn nun auch der durch dieses sonst so vorzügliche gehärtete Filtrirpapier bedingte Fehler meist wohl vernachlässigt werden darf, musste ich für diesmal die Filtration der wässerigen alkalischen Flüssigkeit mit Hülfe von Asbest oder Glaswolle vollziehen. Denn es kommt bei diesen Versuchen auf sehr kleine Unterschiede an, und es handelt sich um eine Frage von grundsätzlicher Bëdeutung.

\section{Reihe I.}

$250 \mathrm{~g}$ frischer Pferdefleischbrei in einen 500 ccm-Kolben von Jenaer Glas gebracht und hinzugegossen $250 \mathrm{ccm}$ Lauge von $60 \%$ $\mathrm{KOH}$. Das Kalipräparat ist das beste im Handel vorkommende, das in der Preisliste von C. A.F. Kall l ba u m aufgeführt ist als Kaliumhýdrat Ia "Merck", das Kilo zu 6,60 Mark.

Die Dauer der Erhitzung betrug 16 Stunden.

Nach Abschluss der Erlitzung wird ein Literkolben mit $500 \mathrm{ccm}$ kohlensäurefreien Wassers gefüllt, letzteres zum Kochen erhitzt und dazu die alkalische Fleischlösung siedend eingegossen und zur Abküblung hingestelit. Durch Asbest filtrirt. Wegen der Langsamkeit der Filtration erhielt ich über Nacht nur

420 cem ganz klarer rother Lösung.

Ich fällte mit $420 \mathrm{~cm}$ Alkohol von $96 \%$ Tr. und decantirte nach einigen Stunden von dem am Boden liegenden Glykogenbrei, den ich dann auf ein Asbestfilter brachte und nach gutem Abtropfen mit einer Flüssigkeit mehrmals wusch, die gleich viel Alkohol und KOH enthält wie die Lösung, aus der das Glykogen ausgeschieden wórdeil war.

Das Glykogen wurde dann in heissem Wasser gelöst und zu $650 \mathrm{ccm}$ aufgefüllt. Hiervon wurden aus immer derselben Bürette

1) Der ehemische Assistent des Bonner physiologisehen Institutes, Herr Dr. Heyer, hat sich aberzeugt, dass durch 10 maliges Auswaschen (Filtration) der gehärteten Faltenfilter das in $15 \%$ iger kalter Kalilauge lösliche Kohlehydrat ausgewaschen werden kann. Um solches Filter dann zu benutzen, müsste das Kali wieder mit Wasser ausgezogen und das Papier getrocknet werden. 
je 100 cem für jeden Versuch abgemessen. $100 \mathrm{cem}$ dieser Glykogenlösung bedürfen zur Neutralisation $2 \mathrm{cem} \mathrm{ClH}$ von $4,4 \%$.

\section{Versuch I.}

Bestimmung des Gehaltes an Glykogen.

$100 \mathrm{ccm}$ Glykogenlösung in $300 \mathrm{ccm}$-Kolben abgemessen.

$+\quad 2 \mathrm{cem} \mathrm{ClH}$ von 4,4\% zur Neutralisation des in der Glykogenlösung enthaltenen Alkalis.

$+100 \mathrm{ccm} \mathrm{ClH}$ von $4,4 \%$.

$+\mathrm{ClH}$ von $2 \%$ q. s.

Invertirung. Nach dieser Invcrtirung ist die Flüssigkeit schwach wie durch feinsten Staub getrübt. Nach Abkühlung und Auffüllung auf $300 \mathrm{ccm}$ mit $\mathrm{ClH}$ von $2 \%$ Filtration durch trockenes schwedisches Filter, Filtrat granz klar und farblos.

$81 \mathrm{ccm}$ der erhaltenen Zuckerlösung liefern:

Analyse 1. $0,1826 \mathrm{Cu}_{2} \mathrm{O}$ (Rohr 4$)=0,1622 \mathrm{Cu}$.

Analyse 2. $0,1816 \mathrm{Cu}_{2} \mathrm{O}$ (Rohr 10).

Die Controle nach Volhard mit Rohr 4 ergibt: 0,1597 Cu $=0,074$ Zucker.

Also

100 cem Glykogenlösung $=0,274 \mathrm{~g}$ Zucker.

\section{Versuch II.}

Bestimmung des Gehaltes an Glykogen, das diesmal mit Alkohol vor der Invertirung gefält wird.

100 cem Glykogenlösung in's Becherglas abgemessen und auf $2 \% \mathrm{KOH}$ gebracht.

200 cem Alkohol $96 \%$ Tr.

Glykogen durch schwedisches Filter abfiltrirt, in $300 \mathrm{ccm}-\mathrm{Kolben}$ nach Neutralisation gebracht, beinahe mit Wasser und ClH aufgefült, so dass die Lösung $2,2 \% \mathrm{ClH}$ enthält. Nach Inversion Verfahren wie bei dem vorherigen Versuch.

81 ecm der Zuckerlösung liefern:

Analyse 1. $0,1773 \mathrm{Cu}_{2} \mathrm{O}$ (Rohr 3) $=0,1574 \mathrm{Cu}$.

Analyse 2. 0,1779 $\mathrm{Cu}_{2} \mathrm{O}$ (Rohr 12).

Die Controle nach Volhard ergab mit Rohr 3

$0,1591 \mathrm{Cu}=0,07365$ Zucker.

Also :

$100 \mathrm{ccm}$ Glykogenlösung $=0,273 \mathrm{~g}$ Zucker, 
Dieser Versuch beweist noch, dass aus einer $2 \%$ Kali enthaltenden Lösung das Glykogen durch 2 Volumina Alkohol von $96 \%$ Tr. vollständig ausgefälltwird.

\section{Versuch III.}

$100 \mathrm{ccm}$ Glykogenlösung $+4 \mathrm{~g}$. $\mathrm{KOH}(6,6 \mathrm{cem}$ Lauge von $60 \% \mathrm{KOH})+$ aq. q. s. in's 200 cem-Kölbchen, 20 Stunden erhitzt. -

Nach Abkühlung im 200 cem-Kölbchen mit $\mathrm{ClH}$ von 1,19 sp. Gew, genau neutralisirt, dann in ein 300 ccm-Kölbchen gegossen und letzteres mit dem Spülwasser aus dem $200 \mathrm{ccm}$-Kölbchen $+15 \mathrm{ccm} \mathrm{ClH}$ von $1,19=6,6 \mathrm{~g} \mathrm{ClH}$ fast aufgefült. Nach Inversion und Filtration durch trockenes schwedisches Filter liefern 81 cem der Zuckerlösung:

Analyse 1. $0,1850 \mathrm{Cu}_{2} \mathrm{O}($ Rohr 3$)=0,1642 \mathrm{Cu}$.

Analyse 2. $0,1854 \quad \mathrm{Cu}_{2} \mathrm{O}$ (Rohr 12).

Die Controle für Rohr 3 nach Volhard liefert:

$$
0,1591 \mathrm{Cu}=0,0737 \text { Zucker. }
$$

Also :

$100 \mathrm{ccm}$ Glykogenlösung $=0,273 \mathrm{~g}$ Zucker.

\section{Versuch IV.}

Dieser Versuch wurde genau so wie Versuch III ausgeführt. Nur habe ich das Glykogen vor der Inversion erst mit Alkohol gefällt. Das $200 \mathrm{ccm}-\mathrm{Fläschchen} \mathrm{wurde} \mathrm{entleert,} \mathrm{ausgespült} \mathrm{und} \mathrm{die} \mathrm{Flüssig-}$ keit auf $300 \mathrm{ccm}$ gebracht, der so viel $\mathrm{KOH}$ zugesetzt worden war, dass sie $3 \% \mathrm{KOH}$ enthielt. Gefällt mit $300 \mathrm{cem}$ Alkohol von $96 \%$ Tr. - Das Glykogen wird abfiltrirt und im $300 \mathrm{cem}-K o ̈ l b c h e n$ invertirt, wie es vorher bei Versuch II dieser Reihe ausgeführt worden ist.

$81 \mathrm{ccm}$ der Zuckerlösung liefern:

Analyse 1. $0,1797 \mathrm{Cu}_{2} \mathrm{O}=0,1598 \mathrm{Cu}$ (Rohr 4).

Analyse 2. $0,1784 \mathrm{Cu}_{2} \mathrm{O}$ (Rohr 10).

Die Controle nach Volhard ergab mit Rohr 4:

$$
0,1553 \mathrm{Cu}=0,0718 \mathrm{~g} \text { Zucker. }
$$

Also :

$100 \mathrm{ccm}$ Glykogenlösung $=0,266 \mathrm{~g}$ Zucker. 
Ergebnisse der Reihe I.

Vergleichen wir die am nächsten vergleichbaren Versuche

Versuch I. Gehalt der Glykogenlösung vor Kochen hnit Kali $=0,274 \%$ Zucker.

Versuch III. Gehalt der Glykogenlösung nach 20 stündigem Kochen mit $2 \%$ Kali $=0,273 \%$ Zucker.

Verlust $0,4 \%$ :

Versuch II. Gehalt der Glykogenlösung vor Kochen mit Kali, aber Fällung des Glykogenes mit Alkoboł $=0,273 \%$.

Versuch IV. Gehalt der Glykogenlösung nach 20 stündigem Kochen mit Kali, aber Fällung des Glykogenes mit Alkohol $=0,266 \%$.

Verlust $=2,6 \%$.

Es wird zweckmässig sein, das Ergebniss der folgenden Reihe kennen zu lernen, ehe wir zu einer Beurtheilung der Versuche schreiten.

\section{Reihe. II.}

$250 \mathrm{~g}$ frischer Pferdefleischbrei in $500 \mathrm{ccm}-$ Kolben von Jenaer Glas; hinzugefügt $250 \mathrm{~cm}$ kalter. Lauge von $60 \% \mathrm{KOH}$. Flasche, mit Uhrglas bedeckt, in's Wasserbad, das sofort erhitzt wird. Erhitzung dauert 16 Stunden.

In einem Literkolben werden $500 \mathrm{ccm}$ Wasser gekocht und "die noch heisse Fleischlösung hinzugegossen, abgekühlt, dụch Glaswollę mit Beihülfe der Saugpumpe filtrirt. Das Filtrat ist trübe.

Ich nehme 800 cem Filtrat

800 ecm Alkohol von $96 \% \mathrm{Tr}$.

Nach Absetzen des Niederschlages giesse ich die vollkommen klare Flüssigkeit $a b$ und filtrire den Niederschlag durch Glaswolle ohne Saugen. Das Filtrat ist anfangs trüb; abermals auf das Filter gegossen wird dasselbe sonnenklar. Nach gutem Abtropfen und Auswaschen mit alkalischem Weingeist löse ich den Glykogenklumpen in siedendem Wasser.

Die trübe Brühe des Filtrates. wurde auf $500 \mathrm{~cm}$ mit sterilisirtem Wasser aufgefüllt und durch schwedisches Filter filtrirt. Das Filtrat zeigt weisse Opalescenz; auf dem.Papier bleibt grünliche Schmiere. 
$500 \mathrm{cem}$ dieses Filtrates werden durch Zusatz von $15 \mathrm{~g} \mathrm{KOH}$, d. h. von $25 \mathrm{ccm}$ meiner Lauge von $60 \% \mathrm{KOH}$, auf $3 \% \mathrm{KOH}$ gebracht und mit $500 \mathrm{cem}$ Alkohol von $96 \%$ Tr. gefällt. Das Glykogen scheidet sich pulvrig, flockig und schön weiss aus. Nachdem die Flüssigkeit mehrere Tage gestanden, ist sie sonnenklar und läsșt sich fast ganz so abgiessen, dass das weisse, weiche Glykogen auf dem Boden des Becherglases liegen bleibt. Nach gutem Abtropfen löse ich im Becherglase mit sterilisirtem Wạsser zu $550 \mathrm{~cm}$; mit denen folgende Versuche ausgeführt werden.

\section{Versuch I.}

Bestimnung des Gehaltes an Glykogen durch unmittelbare Inversion ohne vorherige nochmalige Fällung. des Glykogenes mit Alkohol.

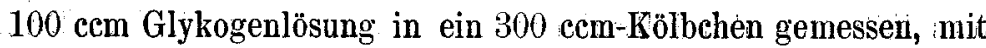
Wasser q. s. und $\mathrm{ClH}$ so aufgefüllt, dass diè Lösung $2,2 \%$ ClH enthält. Nach Inversion abgekühlt, auf 300 cem genau aufgefüllt. $81 \mathrm{cem}$ dieser Zuckerlösung lieferten:

$$
0,2608 \mathrm{~g} \mathrm{Cu}_{2} \mathrm{O}=0,2317 \mathrm{Cu}(\operatorname{Rohr} 4) \text {. }
$$

Die Controle nach Volhard lieferte:

$$
0,2319 \mathrm{Cu}=0,1099 \text { Zucker. }
$$

Also:

$$
100 \mathrm{ccm} \text { Glykogenlösung }=0,407 \mathrm{~g} \text { Zucker. }
$$

Der Versuch wurde wiederholt mit einem anderen Asbestfilterröhrehen (Nr. 10), und es wurde gefunden nach Volhard:

$$
0,2315 \mathrm{Cu}=0,1097 \text { Zucker. }
$$

Also :

$100 \mathrm{cem}$ Glykogenlösung $=0,4052$ Zucker.

Mittel: $100 \mathrm{cem}$ Glykogenlösung $=0,406$ Zucker.

\section{Versueh II.}

Der vorhergehende Versuch hat den Gehalt an Kohlehydrat in $100 \mathrm{ccm}$ Lösung festgestellt. Es frąt sich, ob das ganze Glykogen mit einem gleichen Volum Alkohol von $96 \%$ Tr. bei Gegenwart von $4 \% \mathrm{KOH}$ abgeschieden werden kann.

$100 \mathrm{ccm}$ Glykogenlösung,

$6,6 \mathrm{ccm}$ Lauge von $60 \% \mathrm{HOH}$, $110 \mathrm{ccm}$ Alkohol von $96 \% \mathrm{Tr}$. 
Nach 24 Stunden durch schwedisches Filter filtrirt. Glykogen bleibt fast ganz im Becherglas. Nach Abtropfen der weingeistigen Flüssigkeit wird das das Glykogen noch enthaltende Becherglas unter den Trichter gebracht und dieser mit Wasser aufgefullt, so dass das abfliessende Filtrat das Glykogen im Becherglas löst. Nun wirft man ein kleines Stückehen Lackmuspapier in die Flüssigkeit und lässt aus einer Bürette $\mathrm{ClH}$ (von 1,19) hinzufliessen, bis Neutralität erreicht ist. Nöthig waren $0,35 \mathrm{ccm} \mathrm{ClH.} \mathrm{Diese} \mathrm{neutrale}$ Lösung wird nun in einen $300 \mathrm{ccm}-K_{0}$ lben eingeführt mit Hülfe eines Trichters und hinzugefügt $6,6 \mathrm{~g} \mathrm{ClH}=15 \mathrm{~cm} \mathrm{ClH}$ von 1,19 spec. Gew. - Darauf schiehe ich den $300 \mathrm{ccm}-K o l b e n$ mit dem aufgesteckten Trichter unter das Abflussrobr des das Filter tragenden Trichters und giesse so lange Wasser auf, bis der $300 \mathrm{ccm}-$ Kolben beinahe bis zur Marke gefüllt ist. Zuletzt überzeuge ich mich, dass das Filter an das Filtrationswasser nichts mehr abgibt. Denn Alkohol bringt keine Spur einer Trübung mehr hervor in dem Filtrate.

Nach ausgeführter Inversion fand ich in $81 \mathrm{ccm}$ :

$0,2611 \mathrm{~g} \mathrm{Cu}_{2} \mathrm{O}=0,232 \mathrm{Cu}=0,110 \mathrm{~g}$ Zucker.

Die Controle nach Volhard ergab:

$$
0,2311 \mathrm{Cu}=0,1095 \mathrm{~g} \text { Zucker. }
$$

Also:

$100 \mathrm{ccm}$ Glykogenlösung $=0,4056 \mathrm{~g}$ Zucker.

Diese Versuche sind noch desshalb von besonderer Wichtigkeit, weil sie zeigen, dass ein gleiches Volum Alkohol von $96 \%$ Tr. das Glykogen ans einer wässerigen Lösung von $4 \%$. KOH vollständig ausfällt.

\section{Versueb III.}

$100 \mathrm{ccm}$ Glykogenlösung abgemessen in ein $200 \mathrm{ccm}-K o ̈ l b c h e n$, $6,6 \mathrm{ccm}$ Lauge von $60 \% \mathrm{KOH}$,

Wasser q. s., um das Volum beinahe auf $200 \mathrm{ccm}$ zu bringen.

Die Lösung enthält vor dem Koehen annähernd $2 \% \mathrm{KOH}$. Das Kochen wird 191/2 Stunde in Wasserbad fortgesetzt, abgekühlt, etwas Reagenspapier in das $200 \mathrm{ccm}-\mathrm{Kölbchen,} \mathrm{neutralisirt} \mathrm{mit} \mathrm{ClH}$ von 1,19. Dann ausgegossen in einen $300 \mathrm{ccm}-K o l b e n$, das $200 \mathrm{ccm}-$ Kölbchen mit $15 \mathrm{ccm} \mathrm{ClH}$ von 1,19 ausgespült und in den $300 \mathrm{ccm}-$ Kolben nachgefüllt, dann mit Wasser gespült, bis der $300 \mathrm{ccm}-K o l b e n$ fast bis zur Marke gefüllt ist. Wenn man so verfährt, ist man vor 
Ueber die Einwirkung verdünnter Kalilauge auf Glykogen bei $100^{\circ} \mathrm{C} .85$

jedem Verlust gesichert. Nach Invertirung lieferten $81 \mathrm{ccm}$ der Zuckerlösung:

$$
0,2585 \mathrm{~g} \mathrm{Cu}_{2} \mathrm{O}=0,2296 \mathrm{~g} \mathrm{Cu}(\text { Rohr } 10) .
$$

Die Controle nach Volhard ergab:

$$
0,2286 \mathrm{~g} \mathrm{Cu}=0,1082 \mathrm{~g} \text { Zucker. }
$$

Die Wiederholung ergab:

$$
0,2595 \mathrm{~g} \mathrm{Cu}_{2} \mathrm{O}=0,2304 \mathrm{~g} \mathrm{Cu}(\text { Rohr } 4) \text {. }
$$

Die Controle nach Volhard $=0,2286$.

Also $81 \mathrm{cem}$ Lösung $=108,23 \mathrm{mg}$ Zucker und $100 \mathrm{ccm}$ Glykogenlösung $=0,401 \mathrm{~g}$ Zucker.

\section{Versuch IV.}

Genau wie der vorhergehende Versuch angestellt, d. h. dieselhe Menge Glykogen wurde $19^{1 / 2}$ Stunde in Kalilauge von $2 \%$ im Wasserbad erhitzt.

Diesmal soll aber das Glykogen erst durch Alkohol gefällt werden. Die Lösung enthält bereits $4 \mathrm{~g} \mathrm{KOH}$; da sie des Spülens halber auf $300 \mathrm{ccm}$ gebracht wird und $4 \% \mathrm{KOH}$ enthalten soll, wurden noch $8 \mathrm{~g}$ KOH $=13,3 \mathrm{ccm}$ Lauge von $60 \%$ binzugefügt und die erbaltenen $300 \mathrm{ccm}$ mit $300 \mathrm{cem}$ Alkohol von $96 \% \mathrm{Tr}$. gefällt. Fällt nicht flockig; weiss wie Milch, wie durch feinsten Staub getrübt. Allmählich hat sich das Glykogen als weiche, weisse, flockige Masse zu Boden gesenkt. Einiges sitzt aber am Glase. Nach 24 Stunden vollkommene Klärung. Filtrirt auch ohne Spur von Trübung durch das schwedische Filter. Fast alles Glykogen bleibt nach Abgiessen der Flüssigkeit im Becherglase. Um nun ohne Verlust das Glykogen in den $300 \mathrm{ccm}-K o l b e n$ zu bringen und mit $2,2 \% \mathrm{ClH}$ im Wasserbad zu erhitzen, verfuhr jeh genau so, wie es bei Versuch II beschrieben ist.

$81 \mathrm{ccm}$ Zuckerlösung lieferten:

$$
0,2554 \mathrm{Cu}_{2} \mathrm{O}=0,2269 \mathrm{Cu} \text {. }
$$

Die Controle nach Volhard ergab

Also:

$$
0,22661 \mathrm{Cu}=0,1072 \mathrm{~g} \text { Zucker. }
$$

100 cenl Glykogenlösung $=0,397 \mathrm{~g}$ Zucker.

Ergebnisse.

Vergleichen wir vorerst die Versuche, bei denen das Glykogen vor der Invertirung nicht mit Alkohol gefällt wurde, so dass der Gehalt an Kohlehydrat ohne Verlust zu Tage treten muss, so haben wir; 
Versuch I: Gehalt ohne Kochen mit Kali $0,406 \%$ Zucker, Versuch III: Gehalt nach dẻm Kochen mit : $2 \%$ Kali $0,401 \%$ Zucker.

$$
\text { Verlust }=1,2 \% \text {. }
$$

Vergleichen wir ferner die zwei Versuche, bei denen das Glykogen vor der Invertirung mit Alkohol gefällt wurde, so ergibt sich:

Versuch II: Gehalt an Glykogen ohne Kochen.mit KOH $=0,4056 \%$ Zucker.

Versuch IV: Gehalt an Glykogen wach Kochen mit $2 \%_{0}$ $\mathrm{KOH}=0,3970 \%$ Zucker.

Verlust $=2,1 \%$.

(Siehe Tabelle S. 87.)

\section{Reihe III.}

Die bisher mitgetheilten zwei Versuchsreihen waren in der :Apt ausgeführt worden; dass der kalte Fleichbrei in die kalte Kalilatuge eingetragen und das die Mischung enthaltende Gefäss dann in das siedende Wasserbad getaucht wurde. Es war nicht updenkbar, dass wegen des langsamen Stejgens der Temperatur eine Veränderung des Glykogenes sich vollzog, die in der Bildung von Glykogen-Dextrìn ihren Ausdruck fand. F. W. P a v y behauptet aber, diss Dextrin von Kalilauge angegriffen wird. Desshalb machte ich noch Reihe III und verfuhr folgendermaassen.

$500 \mathrm{ccm}$ Lauge von $60 \% \mathrm{KOH}$ (Marke $\mathrm{I}^{\text {a }}$ "Merek ${ }^{4}$ ) wurden in éinem Literkolben auf $100^{\circ} \mathrm{C}$. erhitzt, sodann in Klümpchen all mählich $500 \mathrm{~g}$ ganz friseher Fleichbrei eingetragen. Fortwährend wird die Flüssigkeit umgeschwenkt, damit sich die Fleischtheilchen möglichst vertheilen. Weil die Flasche auf einem Drahtnetz; uber einer kleinen Flamme steht, ist es möglich, zu verhindern, dass das kalte Fleisch die Temperatur der Flüssigkeit stärker herabdrücke. Mit einem Thermometer controlirte ich fortwährend und fand, dass die Temperatur von $92^{\circ}$ bis $110^{\circ}$ C. schwankte. Nachdem die $500 \mathrm{~g}$ Fleischbrei eingeführt waren, ergab sich, dass sehon fast Alles gelöst war. Gleichwohl erhitzte ich im siedenden Wasserbad noch 2 Stunden.

In einem Kolben von 2 Liter Gehalt brachte ich 300 echr: Wasser zum Kochen, goss die siedende Fleischlösung hinein und spülte dẹn 
Ueber die Einwirkung verdünnter: Kalilauge auf Glykogen bei $100^{\circ} \mathrm{C}$.

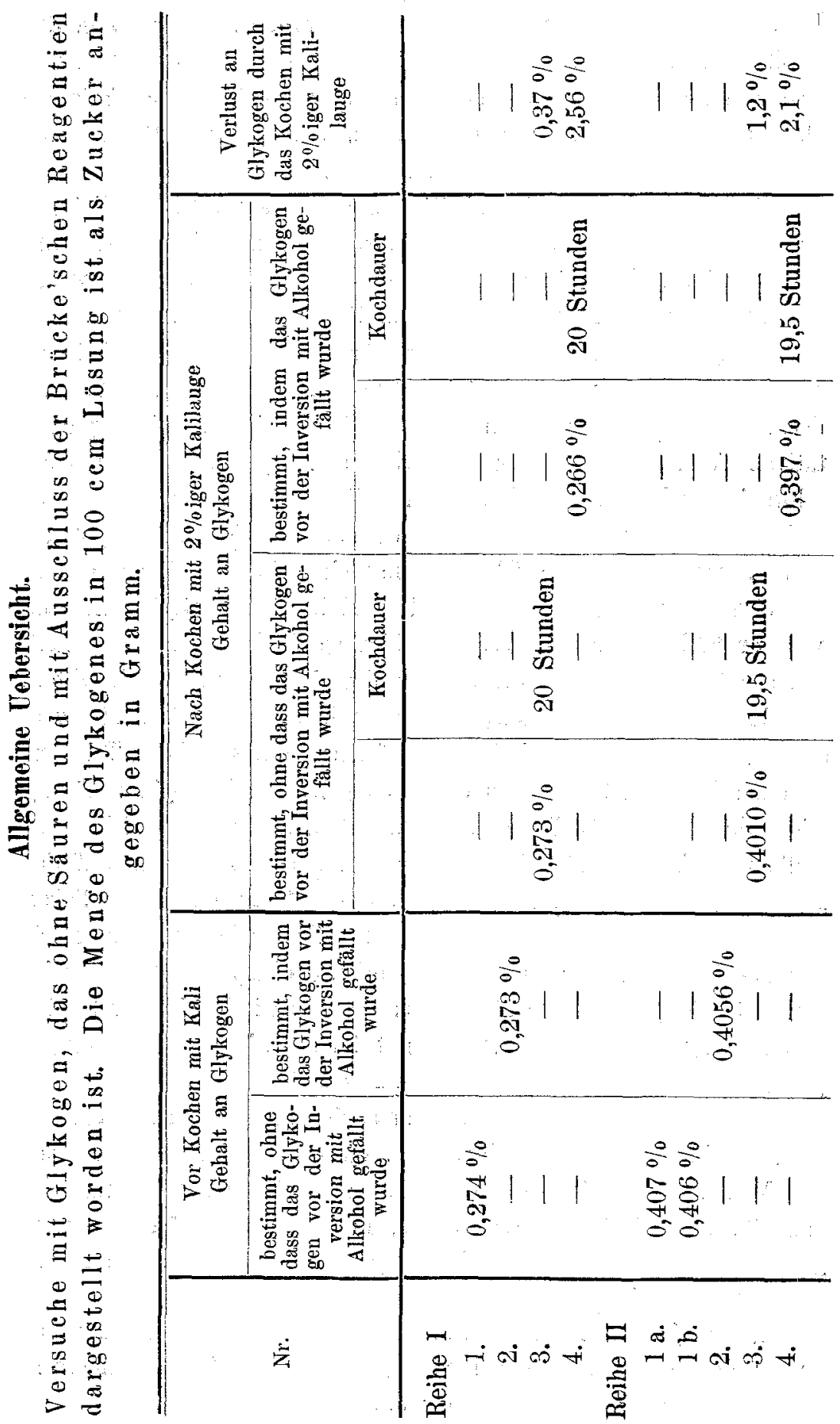


Literkolben mit $200 \mathrm{ccm}$ siedenden Wassers aus, das auch in den 2 Literkolben nachgefült wurde. Nach Abkühlung wird mit sterilisirtem Wasser aufgefüllt und dann die Flüssigkeit zur Erzielung gleichmässiger Mischung in ein grosses Becherglas entleert. Die Mischung enthielt einen sehr feinen Staub.

Das Filtrat, welches der mit Glaswolle beschickte Trichter lieferte, war anfangs ein wenig getrübt, wurde aber zurückgegossen vollkommen klar. Da die Filtration langsam von statten ging, waren 10 Stunden nöthig, bis ich $300 \mathrm{ccm}$ Filtrat gewonnen hatte. Ich fällte mit $300 \mathrm{ccm}$ Alkohol von $96 \% \mathrm{Tr}$. Grobflockige Ausscheidung erfolgte, die sich rasch absetzte. $2^{1 / 2}$ Stunden nach der Fällung decantirte ich fast ohne Verlust, da es sich ja nicht um einen quantitativen Versuch handelte, und filtrirte den Glykogenbrei durch ein schwedisches Filter. Die Filtration vollzog sich schnell, und das rothe Filtrat ist vollkommen klar. Nachdem die Flüssigkeit gut abgetropft war, wurde das Glykogen mit einer Flüssigkeit gewaschen, die eben so viel Kali und Alkohol enthielt wie die Glykogenlösung vor der Fällung. Auch diese Waschlösung filtrirte ziemlich schnell. Nach gutem Abtropfen wurde über das Abflussrohr des Trichters ein Gummischlauch gezogen, den ich mit einem Quetschhahn verschloss. Darauf goss ich kaltes, sterilisirtes Wasser auf den Trichter. In einer Stunde ist fast alles Glykogen gelöst. Die Glykogenlösung wird darauf nach Oeffnung des Quetschhabns in einen. $500 \mathrm{ccm}-$ Kolben filtrirt und sterilisirtes Wasser immer auf's Neue auf das Filter gegossen. Es hinterbleibt nur die Spur eines grünlichen Hauches auf dem Papier. Das zuletzt abfliessende Filtrat gibt mit Alkohol keine Trübung mehr. Die erhaltene Glykogenlösung zeigt starke Opalescenz. In zwei Reagensgläser wird je eine Probe der Lösung eingefüllt, angesäuert und mit Brücke'schem Reagens geprüft. Nicht die Spur einer Trübung!

Nicht einmal ein Unterschied der beiden Proben ist zu sehen, obwohl die eine mit Brücke's Reagens versetzt ist und die andere nicht.

Weil es sich bei diesen Versuchen um sehr kleine Unterschiede handelt, die, wie ich überzeugt bin, nur mit meiner Methode festgestellt werden können, habe ich ferner einige Vorsichtsmaassregeln angewandt, die ich genauer angeben will.

1. $\mathrm{Zu}$ jedem Versuche wurden $100 \mathrm{ccm}$ Glykogenlösung aus immer derselben Bürette bei voll geöffnetem Hahne abgemessen, und zwar in derselben Stunde für alle Versuche. 
2. Wenn man $100 \mathrm{ccm}$ Glykogenlösung unmittelbar in einen $300 \mathrm{cem}-K o l b e n$ einfliessen lässt, sofort mit $\mathrm{ClH}$ invertirt und den: Zucker nach meiner Methode bestimmt, erfährt man den Gehalt an Kohlehydrat ganz genau. -

Wenn man aber die abgemessenen $100 \mathrm{ccm}$ Glykogenlösung erst mit Alkohol fällt, das gefällte Glykogen abfiltrirt, dann wieder löst, invertirt und den Zucker bestimmt, müssen kleine Verluste eintreten, die nicht zu vermeiden sind. Diese Verluste sind wesent-: lich bedingt durch Spuren von Glykogen, welche durch die besten Filter gehen, durch nicht vollständige Fällbarkeit des Glykogenes durch den angewandten Weingeist, weil neben dem Glykogen ein wenig Glykogendextrin vorhanden sein kann, und endlich weil absolutes Auswaschen des Filters und der Gefässwände kaum zu erzielen ist. Bei sorgfältigstem Arbeiten pflegt der Verlust ein Milligramm selten zu übersteigen, wenn man es nur mit normalem Glykogen zu thun hat. - Man kann desshalb nur Versuche vergleichen, bei denen die Alkoholfällung entweder in Anwendung gezogen wurde oder ganz unterblieb.

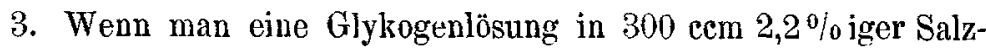
säure invertirt, so hat die Dauer des Kochens einen Einfluss auf die Menge des sich abscheidenden Kupferoxydules. Denn bei länger als 3 bis 5 Stunden fortgesetzter Erhitzung nimmt die Ausbeute an Zucker ab. Wahrscheinlich hat auch die Höhe der Temperatur einen Einfluss. Ich habe desshalb, wenn es irgend möglich war, die beiden Flaschen, deren Glykogenlösungen zu vergleichen waren, gleichzeitig in demselben Wasserbad genau 3 Stunden lang erhitzt.

4. Bei der Bestimmung des Zuckers nach meiner gravimetrischen Methode hat man zu bedenken, dass die Ausbente an Kupferoxydul abhängt sowohl von der Dauer der Erhitzung als auch von der Höhe der angewandten Temperatur. Das in demselben Kessel kochende Wasser hat aber aus verschiedenen Gründen nicht immer dieselbe Temperatur. Desshalb bringe ich in die beiden Bechergläser die beiden verschiedenen Zuckerlösungen, welche mit einander verglichen werden sollen, und erhitze sie in demselben Bade gleichzeitig und gleich lange.

Dadurch verliert man den Vortheil, welcher verbürgt wird, wenn in beiden Bechergläsern gleichzeitig dieselbe Zuckerlösung erhitzt wird. Denn die beiden Analysen müssen denselben Werth geben. Indem man sich dieses Vortheiles begibt, muss man unbedingt für 
das Kupferoxydul nur solche Asbestfilterröhrchen benutzen, welche durch häufige Prüfung als zuverlässig sich bewährt haben.. Die Erfahrung hat mich aber belehrt, dass diese Röhrchen niemals als absolut dicht angesehen werden dürfen, so dass jedes, auch das beste, mit einem kleinen Fehler behaftet ist. Um nicht getäuscht zu werden, mache ich, wo es sich um sehr kleine Unterschiede handelt, desshalb 2 Doppelversuche so, dass im ersten Doppelversuch das der Zuckerlösung $\alpha$ entsprechende Kupferoxydul durch das Asbeströhrchen $A$, das der Zuckerlösung $\beta$ entsprechende Kupferoxydul durch das Asbeströhrchen $B$ filtrirt wird. - In dem zweiten Doppelversuch wird das der. Zuckerlösung $\beta$ entsprechende Kupferoxydul durch Asbestrohr $A$, das der Zuckerlösung $\alpha$ entsprechende Kupferoxydul durch das Asbestrohr $B$ filtrirt. Die Mittel aus beiden $A$-Versuchen und $B$-Versuchen geben dann den richtigen Werth.

Ich habe bei diesen Versuchen die Controle nach Volhard nie versäumt. Sie hat sich als durchaus nothwendig ergeben, wo es sich um kleine Unterschiede handelt.

\section{Versuch I.}

Gebaltsbestimmung der Glykogenlösung.

$100 \mathrm{ccm}$ Glykogenlösung in ein $300 \mathrm{ccm}-$ Kölbchen gemessen, $0,6 \mathrm{ccm} \mathrm{ClH}$ von 1,19 spec. Gew. zum Neutralisiren hinzugegeben $+15 \mathrm{cem} \mathrm{ClH}$ von $1,19+$ Wasser q. s. - Beim Auffüllen auf nahezu $300 \mathrm{ccm}$ enthält die Lösung $2,2 \% \mathrm{ClH}$. - Nach vollzogener Invertirung ist die Flüssigkeit schwach gleichmässig getrübt. Sobald sie sich vollkommen abgekühlt hat, wird genau auf $300 \mathrm{ccm}$ mit Wasser aufgefült und durch schwedisches Filter gegossen. Das Filtrat ist ganz klar und farblos.

$81 \mathrm{ccm}$ Zuckerlösung lieferten:

$$
0,1977 \mathrm{~g} \mathrm{Cu}_{2} \mathrm{O}=\mathbf{0 , 1 7 5 6} \mathrm{Cu} \text { (Rohr 4). }
$$

Nach Volhard's Controle:

$$
0,1753 \mathrm{Cu} \text {. }
$$

Es ist bemerkenswerth, dass vollkommene Uebereinstimmung zischen der gravimetrischen und titrimetrischen Bestimmung besteht.

Also:

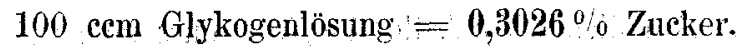


Wiederholung des Versuches 1.

Gefunden:

$0,1979 \mathrm{~g} \mathrm{Cu}_{2} \mathrm{O}=\mathbf{0 , 1 7 5 7} \mathrm{Cu}$ (Rohr 10).

Nach Vollhard's Controle:

$0,1753 \mathrm{Cu}$.

Die Wielerholung gibt denselben Werth, was für die Zuverlässigkeit der Röhrchen 4 und 10 bürgt.

Also:

$100 \mathrm{ccm}$ Glykogenlösung $=\mathbf{0 , 3 0 2 6} \%$ Zucker.

Versuch II.

Glykogenlösung 15 Stunden in $2 \%$ Kalilauge gekocht.

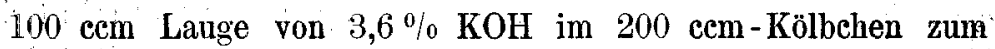
Siederl erhitzt. Hinzugemessen $100 \mathrm{ccm}$ Glykogenlösung. Da letztere eine klèine, bekannte Menge $\mathrm{KOH}$ bereits enthielt, hat man jetzt nahe $200 \mathrm{cem}$ Lösung von $2 \% \mathrm{KOH}$, die 15 Stunden im siedenden Wasserbad erhitzt werden. Hierauf im $200 \mathrm{ccm}-\mathrm{Kölbchen} \mathrm{mit} \mathrm{ClH}$ von 1,19 spec. Gew. neutralisirt. Uebergeführt in einen $300 \mathrm{ccm}-$ Kolben, $15 \mathrm{ccm} \mathrm{ClH}$ von 1,19 spec. Gew. und Wasser fast bis zur Marke eingefüllt, so dass eine Löung von $2,2 \% \mathrm{ClH}$ vorliegt. Nach Invertirung abgekühlt, auf genau $300 \mathrm{ccm}$ mit Wasser aufgefüllt, durch schwedisches Filter die etwas getrübte Flüssigkeit gegossen. Das Filtrat ist klar und farblos.

81 ccm Zuckerlösung ergaben:

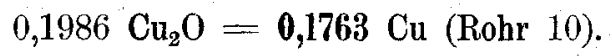

Die Controle nach Volhard:

Also:

$0,1742 \mathrm{Cu}$.

100 ecm Glykogenlösung $=\mathbf{0 , 3 0 0 6}$ Zucker.

Die Wiederholung des Versuches.

$\$ 1 \mathrm{ccm}$ Zuckerlösung ergaben:

$$
0,1993 \mathrm{Cu}_{2} \mathrm{O}=\mathbf{0 , 1 7 6 9} \mathrm{Cu} \text { (Rohr 4). }
$$

Die Controle nach Volbard:

Also:

0,1731 Cu.

$$
\begin{aligned}
\text { Analyse } \mathrm{I} & =0,1742 \mathrm{Cu}, \\
" \quad \mathrm{II} & =0,1731 \mathrm{Cu}, \\
\text { Mitte }] & =0,1736 \mathrm{Cu}=0,0809 \text { Zucker. }
\end{aligned}
$$


Also :

Folglich :

$100 \mathrm{ccm}$ Glykogenlösung $=\mathbf{0 , 2 9 9 6}$ g Zucker.

$100 \mathrm{ccm}$ Glykogenlösung vor Kochen mit Kali $=0,3026$ Zucker 100

$$
\begin{aligned}
& \text { nach " } " 2 \% "=0,2996 \\
& \text { Absoluter Verlust }=0,0030 \text { Zucker. } \\
& \text { Verlust }=0,99 \% .
\end{aligned}
$$

\section{Versuch III.}

Alkoholfällung des Glykogenes:

$100 \mathrm{ccm}$ Glykogenlösung in's Becherglas + $200 \mathrm{ccm}$ Wasser + $19,3 \mathrm{ccm}$ Lauge von $60 \%$. Die Lösung enthält also annähernd $4 \% \mathrm{KOH}$, wenn man beachtet, dass die Glykogenlösung selbst einen kleinen KOH-Gehalt hat. - Gefällt mit $320 \mathrm{ccm}$ Alkohol von $96 \%$ Tr., - nicht flockig, sondern gleichmässig milchig getrübt. - Allmählich setzt sich eine lockere, weiche, weisse, flockige Masse ab. Nur wenig klebt am Glase. Nach 2 tägigem Stehen ganz klar, filtrirt auch klar. In 300 cem-Kolben übergeführt und auf $2,2 \%$ $\mathrm{ClH}$ gebracht. Nach Invertirung filtrirt.

81 ccm Zuckerlösung liefern :

$$
0,1964 \mathrm{Cu}_{2} \mathrm{O}=\mathbf{0 , 1 7 4 4} \mathrm{Cu}(\operatorname{Rohr} 3) \text {. }
$$

Controle nach Volhard:

$$
0,1723 \mathrm{Cu} \text {. }
$$

Wiederholung des Versuches.

81 ccm liefern:

$$
0,1948 \mathrm{Cu}_{2} \mathrm{O}=0,173 \mathrm{Cu}(\text { Rohr 12). }
$$

Controle nach Volhard:

$$
0,1727 .
$$

Also :

$$
\begin{aligned}
\text { Analyse } \mathrm{I} & :=0,1723, \\
\Rightarrow \mathrm{II} & =0,1727, \\
\text { Mittel } & =0,1725=0,0803 \text { Zucker. }
\end{aligned}
$$

Also :

$100 \mathrm{ccm}$ Glykogenlösung $=\mathbf{0 , 2 9 7 4}$ Zucker.

\section{Versuch IV.}

$100 \mathrm{cem}$ Glykogenlösung in $200 \mathrm{ccm}-\mathrm{Kölbchen} \mathrm{mit} 100 \mathrm{ccm}$ Wasser + KOH versetzt, so dass die Lösung $2 \% \mathrm{KOH}$ hat. 
Ueber die Einwirkung verdünnter Kalilauge auf Glykogen bei $100^{\circ} \mathrm{C} .93$

$1 \breve{5}$ Stunden erhitzt. Dann in's Becherglas gegossen, 100 ecm Wasser (Spülwasser) + so viel $\mathrm{KOH}$ hinzugefügt, dass die Lösung auf $4 \%$ $\mathrm{KOH}$ gebracht wird. Gefällt mit einem gleichen Volum Alkohol von $96 \%$ Tr. Abfiltrirt, im 300 ecm-Kolben invertirt; nach Abkühlung auf genau $300 \mathrm{ccm}$ gebracht, filtrirt.

81 cem Zucker liefern:

$$
0,1916 \mathrm{Cu}_{2} \mathrm{O}=0,1703 \mathrm{Cu}(\operatorname{Rohr} 12)
$$

Die Controle nach Volhard:

$0,1691 \mathrm{Cu}$.

Wiederholung des Versuches.

81 cem Zuekerlősung liefern:

$$
0,1908 \mathrm{Cu}_{2} \mathrm{O}=\mathbf{0 , 1 6 9 4} \mathrm{Cu}(\mathrm{Rohr} 3) \text {. }
$$

Die Controle nach Volhard:

$$
0,1696 \mathrm{Cu} \text {. }
$$

Also:

$$
\begin{aligned}
& \text { Analyse I } 0,1691 \mathrm{Cu}, \\
& \Rightarrow \quad \frac{\text { II } 0,1696 \mathrm{Cu},}{0,1693 \mathrm{Cu}}=0,0787 \text { Zucker. }
\end{aligned}
$$

Also:

$100 \mathrm{ccm}$ Glykogenlösung 0,2915 g Zucker.

Versuch III. Vor Kochen mit KOH $=0,2974$ Zucker.

Versuch IV. Nach Kochen mit $2 \% \mathrm{KOH}=0,2915$ Zucker.

Absoluter Verlust $\overline{=0,0059} \mathrm{~g}$ Zucker. Verlust $1,98 \%$.

(Siehe Tabelle auf S. 94.)

Die Reihe III hat wesentlich dieselben Ergebnisse geliefert wie Reihe I und II.

Diese Ergebnisse lassen sich in den Satz zusammenfassen, dass durch das viele Stunden fortgesetzte Kochen einer Glykogenlösung mit verdünnter Kalilauge der Gehalt an Koblehydrat kaum geändert wird. Denn die Verluste schwankten von $0,37 \%$ bis $1,35 \%$. -

Vergleicht man aber das durch Alkohol fällbare Glykogen vor und nach der Kalibehandlung, so steigt der Verlust auf $1,98 \%$ bis $2,56 \%$.

Bei der gewöhnlichen Glykogenanalyse liegen diese Unterschiede im Bereiche der Beobachtungsfehler. Bei der Art, wie ich die Analyse angestellt habe, muss die Thatsache für bewiesen gelten, dass 


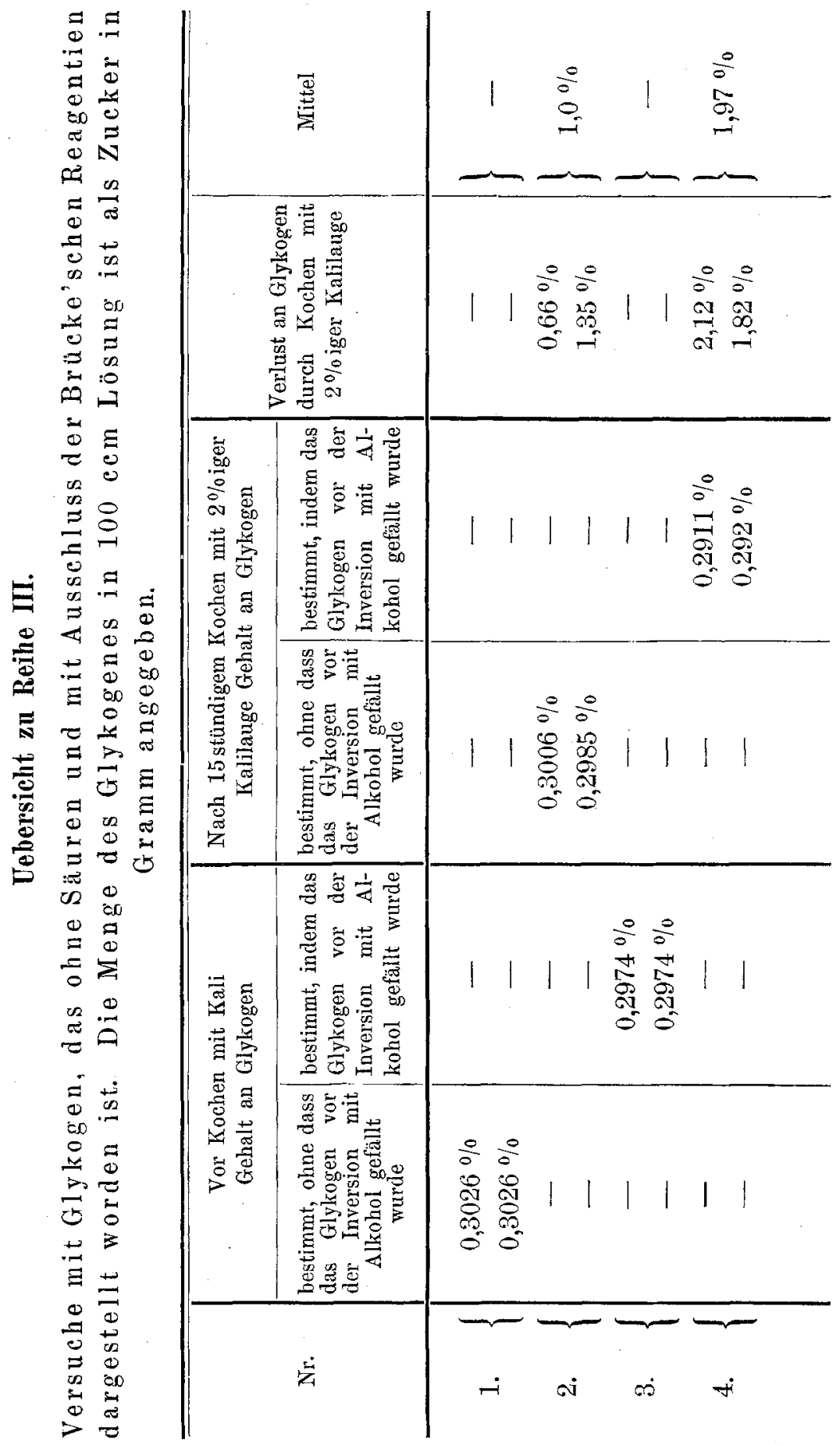


Ueber die Einwirkung verdünnter Kalilange auf Glykogen bei $100^{\circ} \mathrm{C} .95$

das Kochen mit 2\% iger Kalilauge eine kleine Veränderung des Glykogenes bewirkte, die sich in grösserer Löslichkeit in Weingeist aussprach und wahrscheinlich auch mit der Zerstörung einer noch kleineren Menge sich verknüpfte. F. W. Pavy hat die Zerstörbarkeit des Amylodextrins durch Kalilauge dem Amylon gegenüber festgestellt und ist desslaalb der Ansicht, dass auch Glykogendextrin sich äbnlich verhalte. Meine Versuche würden also erst darn streng beweisend sein, wenn festgestellt wäre, dass bei denselben eine geringe Dextrinbildung ausgeschlossen war.

Ich habe alle Vorsichtsmaassregeln so getroffen, um eine Dextrinbildung möglichst zu verhindern. Keine Säure ist mit meinem Glykogen vor der Invertirung in Berührung gekommen, alles angewandte Wasser war sterilisirt, die Glaswolle vor dem Gebrauch ausgekocht u. s. w. - Was ich nicht hindern konnte, war die über die ganzo Nacht sich hinziehende Filtration durch die Glaswolle. Der yrosse Reichthum an gelöstem Eiweiss könnte hier trotz der 15\% $\mathrm{KOH}$ vielleicht einen zu Fermentbildung geeigneten Boden geschaffen haben. Ich kann also mit Sicherheit den vollkommenen Ausschluss von Fermentwirkungen nicht behaupten.

Verfehlen will ich nicht, hervorzuheben, dass F. W. P a y y schon vor mir dieselbe Frage untersuchte und bemerkenswerter Weise Verluste von derselben Grösse wie ich beobachtete. Bei 1/2 stündigem Kochen von Glykogen in Kalilauge von $10 \%$ erhielt F. W. P a v y ${ }^{1}$ ) Verluste von 1,8 bis $2,4 \%$. Das Glykogen war mit der Kalimethode aus der Leber gewonnen worden. -

Trotz alledem sind Thatsachen vorhanden, welche sehr dafüx sprechen, dass das normale Glykogen der Organe auch durch verdünnte Kalilauge von 1 bis $2 \%$ nicht angegriffen wird.

Ich denke hier in erster Linie an mehrere in meinem Laboratorium von Dr. J. Nerking ausgeführte Reihen von Analysen.

In Reihe VI ${ }^{2}$ ) kochte $\operatorname{Er} 250 \mathrm{ccm}$ Fleischlösung von ungefähr $0,8 \% \mathrm{KOH}$ ein Mal 24 Stunden, dann $250 \mathrm{cem}$ derselben Fleischlösung 120 Stunden mit dem Ergebniss:

250 cem Fleischlösung enthielt vor dem Kochen mit Kalilauge 0,3206 g Glykogen.

1) F. W. Pary, The Physiology of the Carbohydrates. An Epicriticism p. 38. London 1895 .

2) Dieses Archiv Bd. 81 S. 21 (1900). 
250 ccm Fleisehlösung nach 24. Stunden Kochen mit 0,8\% Kalilauge $0,3182 \mathrm{~g}$ Glykogen.

$250 \mathrm{ccm}$ Fleischlösung nach 120 Stunden Kochen mit $0,8 \%$ Kalilauge 0,3206 g Glykogen.

Bei Nerking finden sich noch mehr, wenn auch weniger elegante Reihen, welche bezeugen, dass das in den Organen enthaltene Glykogen beliebig lang mit verdünnter Kalilauge erhitzt werden kann, ohne dass es eine Zersetzung erleidet.

Es darf nun allerdings nicht ausser Acht gelassen werden, dass die verdünnte Kalilauge bei der Analyse der Organe vielleicht nur scheinbar das Glykogen unversehrt lässt, weil das Eiweiss das Kali bindet und desshalb das Glykogen schützt. Ich werde diese Frage in meinem Laboratorium sofort weiter verfolgen lassen.

Wie aber auch immer die Wirkung der verdünnten Kalilauge auf Glykogen erklärt werden muss, so steht doch so viel fest, dass die hier vielleicht in Betracht kommenden Fehler sehr kleine sind gegen diejenigen, welche bei dem nach $\mathrm{Brü} c \mathrm{ke}-\mathrm{Külz}$ dargestellten Glykogene bisher beobachtet worden sind.

v. Vintschgall und Dietl ${ }^{1}$ ) fanden: "Wenn man Glykogen „durch längere Zeit (2 bis 3 Stunden) mit Kalilösung (von $1 \%$ bis „3\%) erwärmt, so entsteht ein Verlust, der bis zu $11,7 \%$ steigen "kann."

Richard $\mathrm{Külz} \mathrm{z}^{2}$ erhitzte Glykogen in $1 \%$ iger Kalilösung 1 bis 2 Stunden und hatte Verluste von 4,88 bis $10,52 \%$. Er fällte jedes Mal nach dem Kochen die neutralisirte Lösung mit Alkohol und wog das Glykogen unter Berücksichtigung des Aschengehaltes.

F. W. Pavy hat auch dieser Frage sein Augenmerk zugewandt und kommt zu dem Ergebniss, dass das nach Brücke dargestellte Glykogen eine Veränderung erlitten hat, so dass es der siedenden Kalilauge nicht mehr widersteht. F. W. $\mathrm{Pa} \mathrm{y}^{3}$ ) beobachtete dann Verluste von $19,4 \%$ und noch mehr.

Als ich ${ }^{1}$ ) selbst die Versuche der genannten Forscher mit den von diesen angewandten Methoden wiederholte, aber das Kochen des Glykogenes in $2 \%$ iger Kalilauge viel länger, nämlich 24 Stunden,

1) Dieses Arehiv Bd. 13 S. 261.

2) Zeitschr. f. Biologie Bd. 22 S. 173.

3) F. W. Pavy, The Physiology of the Carbohydrates. An Epicriticism p. 38. London 1895.

4) Dieses Archiv Bd, 75 S. 163. 
fortsetzte, stieg der Verlust auf $45 \%$. - Nach dem Kochen hatte auch ich mit verdünnter Salzsäure neutralisirt und dann mit $2^{1 / 2}$ Vol. Alkohol von 96 Vol.-Procent gefällt. -

In letzter Zeit habe ich diese Versuche mit Benutzung meiner neuen Methoden wiederholt, welche die durch Verunreinigungen bedingten Täuschungen ausschliessen. Bei viele Stunden andauernder Erhitzung des nach Brücke-Külz dargestellten Glykogenes in $2 \%$ iger Kalilauge beobachtete ich einen Verlust von ungefähr $6 \%$ an Kohlehydrat, gemessen durch die Reduction der Kupferoxydlösung. Bestimmte ich den Verlust, wie bisher gebräuchlich, durch Fällung mit zwei Volumina Alkobol von $96 \%$ Tr., so wuchs der Verlust auf ca. $12 \%$. - Durch das Kochen mit verdünter Kalilauge war also ein Theil des Glykogenes nur löslicher in Weingeist geworden - wohl dureh Uebergang in Dextrin -, ein anderer Theil aber war als Kohlehydrat zerstört ${ }^{1}$ ).

Die hier mitgetheilten Thatsachen werden es wünschenswerth erscheinen lassen, dass für die quantitative Analyse des Glykogenes der Organe nicht verdünnte, sondern sehr starke Kalilauge von etwa $30 \%$ in Anwendung kommt.

1) Dieses Archiv Bd. 92 S. 100. 